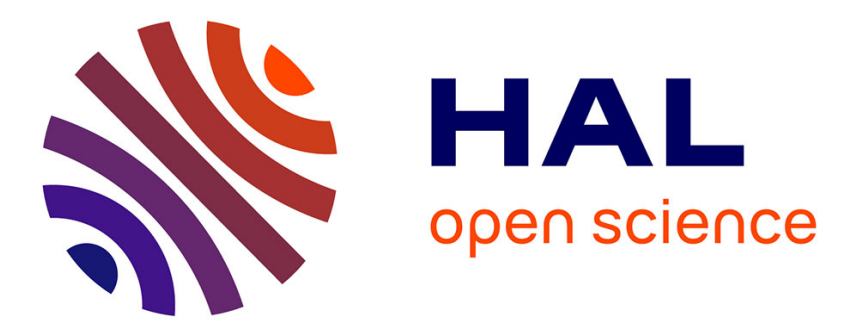

\title{
Lambeau libre de jambe banque pour couverture après hémipelvectomie ou désarticulation de hanche
}

\author{
Steven Roulet, Louis-Romée Le Nail, Gualter Vaz, Antoine Babinet, Valérie
} Dumaine, Aurélie Sallot, Philippe Rosset

\section{- To cite this version:}

Steven Roulet, Louis-Romée Le Nail, Gualter Vaz, Antoine Babinet, Valérie Dumaine, et al.. Lambeau libre de jambe banque pour couverture après hémipelvectomie ou désarticulation de hanche. Revue de Chirurgie Orthopédique et Traumatologique, 2019, 105, pp.22 - 30. 10.1016/j.rcot.2018.12.007 . hal-03486344

\section{HAL Id: hal-03486344 \\ https://hal.science/hal-03486344}

Submitted on 20 Dec 2021

HAL is a multi-disciplinary open access archive for the deposit and dissemination of scientific research documents, whether they are published or not. The documents may come from teaching and research institutions in France or abroad, or from public or private research centers.
L'archive ouverte pluridisciplinaire HAL, est destinée au dépôt et à la diffusion de documents scientifiques de niveau recherche, publiés ou non, émanant des établissements d'enseignement et de recherche français ou étrangers, des laboratoires publics ou privés.

\section{다)(1) $(5$}

Distributed under a Creative Commons Attribution - NonCommerciall 4.0 International 


\section{Mémoire original}

\section{Lambeau libre de jambe banque pour couverture après hémipelvectomie ou} désarticulation de hanche

\section{Free fillet lower leg flap for coverage after hemipelvectomy or hip disarticulation}

Steven Roulet ${ }^{1}$; Louis-Romée Le Nail ${ }^{1,2}$; Gualter Vaz ${ }^{3}$; Antoine Babinet ${ }^{4}$; Valérie

Dumaine $^{4}$; Aurélie Sallot ${ }^{5}$; Philippe Rosset ${ }^{1,2}$

${ }^{1}$ Département de chirurgie orthopédique 2, Centre Hospitalo-Universitaire Tours - Faculté de Médecine, Université de Tours, F-37000 Tours, France

${ }^{2}$ PhyOs, Bone sarcomas and remodeling of calcified tissues - INSERM UN UMR 1238-

Faculté de Médecine de Nantes - F-44000 Nantes, France

${ }^{3}$ Département de chirurgie oncologique, Centre Léon-Bérard, 28 rue Laënnec, F-69008 Lyon, France

${ }^{4}$ Département de chirurgie orthopédique et reconstructive, Centre Hospitalo-Universitaire Cochin- Port Royal, 27 Rue du Faubourg St Jacque F-75014 Paris, France

${ }^{5}$ Département de chirurgie plastique et reconstructive, Centre Hospitalo-Universitaire de Tours - Faculté de Médecine, Université de Tours, F-37000 Tours, France

Auteur correspondant :

Steven ROULET

Département de chirurgie orthopédique 2, Centre Hospitalo-Universitaire Tours, F-37000 Tours, France 
tel : +332474759 15. Fax : +332474783 85. Mail : steven.roulet@orange.fr

Ne pas utiliser, pour citation, la référence française de cet article, mais celle de l'article original paru dans Orthopaedics \&Traumatology: Surgery \& Research, en utilisant le DOI ci-dessus.

\section{Résumé :}

Introduction : La résection carcinologique est le traitement de référence des sarcomes des tissus mous et osseux. Au bassin, cet impératif, peut nécessiter une hémipelvectomie pouvant compromettre la fermeture cutanée de première intention. L'utilisation de lambeaux est indispensable, mais la vascularisation des potentiels lambeaux pédiculés peut avoir été réséquée lors de l'exérèse. Le recours aux tissus sains du membre amputé comme lambeau libre peut constituer une excellente solution de couverture.

Hypothèse : Le lambeau libre de jambe banque est une technique de couverture simple et fiable après hémipelvectomie ou désarticulation de hanche.

Matériel et Méthodes : Sept patients ont été opérés dans 3 centres de références : six désarticulations inter-ilio-abdominales et une désarticulation coxo-fémorale. Le lambeau était constitué dans trois cas de la loge postérieure superficielle du mollet et pour les autres cas de toutes les loges de la jambe avec la fibula non dépériostée. Les différentes complications ont été analysées.

Résultats : Pour tous les patients les marges étaient saines (R0). Le recul moyen lors de la dernière évaluation était de 13 mois (de 6,5 à 21 mois). Au total, six patients ont une complication sur le lambeau avec une seule perte du lambeau. Quatre patients ont pu être appareillés.

Discussion : Le lambeau libre de jambe banque est une technique fiable (86\%) pour la couverture après hémipelvectomie ou désarticulation de hanche. Dans la littérature aucun échec de cicatrisation n'est décrit pour les 16 cas rapportés. Les lambeaux locaux sont souvent trop fragiles et avec un matelassage musculaire insatisfaisant. Ce lambeau libre est à 
privilégier en première intention car il permet d'économiser les autres lambeaux libres potentiels en cas de faillite sans augmenter la morbidité d'un geste déjà important. Cette technique doit faire partie des solutions de couverture après hémipelvectomie.

Niveau de Preuve : IV, étude rétrospective

Mots-clés : lambeau libre ; jambe banque ; hémipelvectomie ; désarticulation inter-ilioabdominale ; désarticulation de hanche

\section{Introduction :}

La résection carcinologique est le traitement de référence des sarcomes des tissus mous et osseux. Le taux de récidive locale varie de 6 à $33 \%$ et dépend du grade histologique et de la qualité des marges de résection [1-5]. Au bassin, cet impératif peut nécessiter une hémipelvectomie externe ou désarticulation inter-ilio-abdominale (DIA) [6], dont la fermeture cutanée, parfois difficile, peut compromettre la réalisation d'une radiothérapie ou d'une chimiothérapie adjuvante du fait de complications. Si la fermeture est impossible de première intention, le recours à un lambeau est indispensable, mais la vascularisation des lambeaux pédiculés peut avoir été réséquée lors de l'exérèse. Un lambeau libre, souvent de grande taille, est alors nécessaire. Afin de préserver les autres lambeaux et en particulier le grand dorsal chez ces malades amenés à solliciter leurs épaules, Workman et al. [7], en 1992, a décrit un cas de lambeau libre comportant les muscles de la jambe du membre amputé. Depuis, dix publications [8-17] ont rapporté au total quinze autres cas. Nous décrivons sept cas de patients présentant un sarcome des tissus mous ou osseux primitif du bassin ou de l'extrémité supérieure du fémur avec une extension locale telle que la résection carcinologique imposait une DIA ou une désarticulation coxo-fémorale (DCF) entrainant un défaut de couverture en 
fin d'intervention. Le but de ce travail était d'évaluer la fiabilité de ce lambeau libre et d'apporter des précisions techniques sur sa réalisation.

\section{Matériel et méthode :}

Les dossiers de sept patients (une femme et six hommes) âgés en moyenne de 54 ans (de 32 à 62 ans), opérés entre 2012 et 2016, dans trois centres références de chirurgie orthopédique oncologique, appartenant au réseau NetSarc-ResOs (netsarc.sarcomabcb.org), ont été analysés (tableau 1). Cinq patients avaient déjà été opérés. Le diagnostic a toujours été posé après une biopsie chirurgicale. Tous les patients présentaient un stade II B de la classification d'Enneking [18], il s'agissait de tumeurs de haut grade avec envahissement extracompartimental sans métastase. Dans deux cas, un traitement par chimiothérapie néoadjuvante a été réalisé. Un bilan d'imagerie comprenant au moins un angio-scanner et/ou une IRM avait permis d'exclure une localisation secondaire au niveau du membre amputé et de s'assurer de la qualité des axes vasculaires. La conservation du membre n'était possible pour aucun des patients. Pour six d'entre eux, le volume de la tumeur et l'envahissement des axes vasculaires interdisaient une chirurgie conservatrice (figure 1). Dans un cas la conservation n'était pas envisageable en raison d'une perte de substance majeure osseuse et des parties molles à prévoir : il s'agissait d'un malade qui avait eu une prothèse de hanche avec alésage fémoral sur fracture pathologique imposant une arthrectomie de hanche monobloc sans possibilité de lambeau pédiculé. Il s'agissait dans six cas d'une DIA et dans un cas d'une DCF avec résection de la région glutéale.

Les malades étaient installés en décubitus latéral avec des appuis thoraciques antérieur et postérieur permettant un roulis de la table vers l'avant et l'arrière (figure 2), sans appui pelvien.

Le lambeau était abordé selon Workman et al. [7] par une incision antérieure sur la crête 
tibiale, par des incisions circonférentielles à la cheville et au creux poplité. Une incision centrée sur la face postérieure de la cuisse permettait la dissection des vaisseaux fémoraux pour obtenir un pédicule long (figure 3). En cas d'utilisation des 4 loges de la jambe, la fibula était raccourcie mais conservée afin de protéger, par sa rigidité, les axes vasculaires du lambeau. Dans 2 cas elle a été fixée aux tranches osseuses par des points de fils résorbables, afin de jouer un rôle de stabilisateur du lambeau. Sa conservation permettait également d'apporter un support à la reconstruction du pelvis et un renfort potentiel pour l'appareillage. Les différents temps chirurgicaux se décomposaient comme suit en fonction des équipes : soit par la même équipe avec dissection du lambeau puis abord pelvien et amputation puis sevrage du lambeau et anastomoses; soit abord pelvien et amputation puis dissection et levée du lambeau sur table; soit en double équipe : abord pelvien et dissection du lambeau en parallèle puis amputation et levée du lambeau. Les anastomoses ont été réalisées par un chirurgien vasculaire (tableau 2). Dans cinq cas le lambeau a été rincé avec du sérum hépariné et dans trois cas une injection intraveineuse d'héparine a été réalisée.

\section{Résultats :}

Le lambeau était constitué dans trois cas de la loge postérieure superficielle du mollet (muscles gastrocnémiens et soléaire) et dans 4 cas de toutes les loges de la jambe avec la fibula non dépériostée (figure 4). Les détails de la chirurgie et des complications post opératoires sont rapportés dans le tableau 2. Sur les sept malades, six ont eu une complication sur le lambeau. Une perte de lambeau (14\%) est à déplorer (tableau 2). Pour un cas (tableau 2, cas $\mathrm{n}^{\circ}$ ), l'artère tibiale antérieure a été lésée lors de la dissection du lambeau Un lambeau a dû être déposé au vingt-et-unième jour, après plusieurs reprises chirurgicales du fait d'une thrombose veineuse malgré une procédure de rinçage du lambeau au sérum hépariné associée 
à l'injection intra-veineuse d'héparine durant l'intervention. Un pansement avec pression négative a permis dans un second temps une couverture par une greffe de peau mince. Un deuxième patient a présenté une thrombose veineuse du lambeau au cinquième jour traitée par héparine à dose curative, puis une infection qui a nécessité une reprise au douzième jour et qui a évolué favorablement sous antibiothérapie. Un autre patient a été réopéré pour une infection locale du lambeau au dix-septième jour avec une évolution favorable sous antibiothérapie. Pour un quatrième patient, une petite zone nécrotique du lambeau a dû être parée chirurgicalement au quinzième jour. Chez un cinquième patient, l'anastomose veineuse a dû être reprise le soir même de l'intervention pour un défaut de suture. Pour deux patients, des complications urologiques sont survenues avec une plaie de l'uretère nécessitant la mise en place d'une sonde $\mathrm{JJ}$ au deuxième jour post-opératoire, et une plaie calicielle nécessitant également une sonde au quinzième jour post-opératoire. Dans un cas, il s'est produit au quatre-vingtième jour une ulcération cutanée sur l'extrémité de la fibula au travers du lambeau nécessitant une reprise chirurgicale pour ostéotomie raccourcissante. Au total, sur les sept malades, six ont eu une complication sur le lambeau mais une seule perte du lambeau est survenue (tableau 2). Aucun problème de hernie n'a été rapporté. Pour un patient, il a été observé à quatre mois post-opératoires, une évolution inattendue avec ossification de l'espace entre la fibula et la symphyse pubienne (figures 5 et 6 ). Sur le plan carcinologique les marges étaient saines (R0) dans tous les cas.

Quatre patients ont pu être appareillés et marchaient grâce une prothèse canadienne avec ou sans béquilles (vidéo 1). Le recul moyen lors de la dernière évaluation était de 13 mois (de 6,5 à 21 mois) (figure 7). Cinq patients ont développé des localisations secondaires : métastases osseuses, pulmonaires et/ou surrénaliennes, et sont décédés suite à leur maladie. La survie moyenne était de 14 mois (de 6,5 à 21 mois). 


\section{Discussion :}

Cette étude multicentrique rapportant 7 cas de lambeau libre de jambe banque démontre que la fiabilité de ce lambeau est importante ( $86 \%$ de succès). Cette série est la plus importante de la littérature qui ne décrit au total que 16 cas, rapportés dans 11 articles [7-17] (tableau 3) et aucun échec dans ces cas décrits.

Après une DIA ou DCF, la cicatrisation doit être obtenue rapidement pour permettre la réalisation du potentiel traitement adjuvant comme une radiothérapie et/ou chimiothérapie, et le site opératoire doit être une zone qui puisse permettre l'adaptation d'un appareillage [912,19-21]. Le fait de ne pas utiliser un lambeau local permet de simplifier la stratégie de résection en s'affranchissant des contraintes de dissection d'un pédicule. Les lambeaux locaux sont souvent trop fragiles $[8,21]$ et avec un matelassage musculaire insuffisant. Ils peuvent présenter jusqu'à $80 \%$ de complications [7,11,19,22,23]. Le lambeau de gluteal maximus [20,24-31] est un lambeau musculo-cutané vascularisé par l'artère glutéale inférieure provenant de l'artère iliaque interne et des artères perforantes d'origine sacrée $[19,23,25,30,32]$. Lorsque son pédicule est conservable, sa dissection est difficile en endopelvien avec un risque de lésion [13,33]. Il est rapporté jusqu'à 55\% de nécrose [7,19,22,30]. Les lambeaux postérieurs fascio-cutanés, sans aucun apport musculaire, ont un risque de nécrose de 80 à 90\% [23]. Les lambeaux utilisant la loge antérieure de la cuisse [19,20,27,30,34-39] sont vascularisés par l'artère circonflexe latérale issue de l'artère fémorale profonde et ne sont donc pas utilisables en cas d'envahissement vasculaire en amont. Leurs complications varient de 37,5 à 67\% des cas [19,30,39]. Le lambeau abdominal : vertical rectus abdominis myocutaneous (VRAM) [11,20,21,40-42] est une solution de couverture à prendre en compte mais la morbidité du site donneur est quant à elle très importante. Il est nécessaire de reconstruire la paroi abdominale avec du matériel synthétique. 
D'autre part, en cas de non conservabilité des vaisseaux épigastriques inférieurs homolatéraux sur lesquels est pédiculé el lambeau, il est nécessaire de prélever le lambeau sur le côté controlatéral, ce qui augmente la morbidité du geste. De plus, il est souvent nécessaire, dans la chirurgie oncologique du bassin, de réaliser des stomies qui rendent ce dernier difficile d'utilisation [20]. En ce qui concerne les lambeaux libres, le latissimus dorsi (LD) est le lambeau habituellement utilisé $[11,20,24,43,44]$ pour couvrir les grandes pertes de substances, mais il expose à une diminution de la force musculaire chez ces patients. En effet, le LD est sollicité pour l'utilisation d'un fauteuil roulant ou des cannes anglaises [7]. La qualité de ces deux lambeaux (VRAM et LD) est également inférieure en terme de vascularisation et de robustesse mécanique par rapport au lambeau de jambe qui apporte une quantité importante de fascia et de muscle $[12,45]$.

En prenant en compte la simplicité technique de levée du lambeau, le fait que la résection carcinologique ne compromette pas sa vascularisation, la qualité du matelassage musculaire qu'il apporte prévenant des hernies de paroi $[7,19,46]$ et l'absence de morbidité du site donneur, le lambeau libre de jambe banque apparaît comme étant une solution très intéressante en cas de lambeau pédiculé de grand fessier ou de quadriceps non réalisable. De plus, sachant qu'en cas d'hémipelvectomie réalisée sur un site chirurgicalement traumatisé le risque de complications (désunion, nécrose, infection) et de récidive locale est plus important [46,47]. Une couverture de bonne qualité est préférable pour les patients déjà opérés à plusieurs reprises notamment en cas de maladie exostosante (tableau 2, cas $n^{\circ} 4$ ). Un autre avantage que présente le lambeau de jambe banque est qu'il n'entame pas le capital de reconstruction et qu'il reste des solutions de couverture en cas d'échec de ce dernier. Il est à privilégier en première intention car il permet d'économiser les autres lambeaux potentiels en cas de faillite sans augmenter la morbidité d'un geste déjà important. Néanmoins, pour être fiable, la réalisation de ce lambeau nécessite le respect de certains 
impératifs techniques. Dans notre étude, les 2 cas de thromboses veineuses du lambeau, dont un à aboutit à un échec, sont survenus lors d'une anastomose veineuse réalisée sur une veine ligaturée. Il paraît primordial de privilégier l'anastomose veineuse termino-latérale sur une veine à débit élevé comme la veine iliaque primitive ou la veine cave en dernier recours, ce qui permet un meilleur drainage veineux. Pour l'anastomose artérielle, il est préférable de ne pas dissocier les artères tibiales antérieure et postérieure comme l'ont réalisé Sara et al. [8] qui rapportent une nécrose de $10 \%$ de la surface du lambeau en réalisant une anastomose entre l'artère tibiale postérieure et l'artère glutéale supérieure, et entre l'artère tibiale antérieure et l'artère épigastrique inférieure.

La dissection du lambeau doit être réalisée en début d'intervention avant la ligature des axes vasculaires et l'amputation pour limiter le temps d'ischémie, comme recommandé par Bibbo et al. [17]. L'utilisation de toutes les loges musculaires de la jambe permet d'apporter un volume musculaire important et les réseaux anastomotiques sécurisent la survenue d'une lésion d'un des axes vasculaires. La conservation de la fibula non dépériostée [10,11] permet de gagner du temps d'intervention, mais surtout elle prévient les lésions de l'axe vasculaire fibulaire qui est en dedans, apporte un support à la reconstruction du pelvis et un renfort pouvant aider pour l'appareillage. Toutefois, nous recommandons de réaliser une ostéotomie distale d'au moins un tiers à la moitié afin d'éviter toute complication à type de fistulisation par perforation du lambeau par le moignon de fibula (cas $\left.n^{\circ} 4\right)$. En proximal, compte tenu de la proximité de l'émergence de l'artère tibiale antérieure au travers de la membrane interosseuse, nous ne recommandons pas d'ostéotomie. La fibula peut être ostéosuturée au sacrum ou au moignon d'ilium. La dissection du pédicule fémoro-poplité doit être étendue en proximal pour avoir une longueur suffisante pour orienter à la demande le lambeau et ne pas être bridé lors de son positionnement pelvien. La confection d'un vêtement compressif postopératoire type bermuda sur mesure permet d'éviter les collections et les séromes rapportés 
dans la littérature [11,39].

\section{Conclusion :}

L'utilisation du lambeau libre de jambe du membre amputé lors d'amputations proximales permet de s'affranchir des difficultés liées à l'utilisation de lambeaux locaux qui s'avère parfois impossible et évite la morbidité des sites donneurs traditionnels à distance. L'apport musculaire important assure un bon matelassage réduisant les complications locales et assure un moignon de belle qualité et donc un appareillage performant. Cette technique de couverture est fiable et simple mais requiert une équipe chirurgicale pluridisciplinaire entrainée. Elle doit faire partie des solutions de couverture après hémipelvectomie.

Conflit d'intérêt : Aucun auteur ne déclare de conflit d'intérêt.

Financement : Aucun financement n'a été reçu

Contribution des auteurs :

SR : collecte d'information et écriture

Les autres coauteurs : collecte information et relecture

\section{Références :}

1. Anract P, Biau D, Babinet A, Tomeno B. Pelvic reconstructions after bone tumor resection. Bull Cancer (Paris) 2014;101:184-194.

2. Anderson ME. Update on survival in osteosarcoma. Orthop Clin North Am. 2016;47:283-292

3. Maretty-Nielsen K, Aggerholm-Pedersen N, Safwat A, et al. Prognostic factors for local recurrence and mortality in adult soft tissue sarcoma of the extremities and trunk wall: a cohort study of 922 consecutive patients. Acta Orthop. 2014;85:323-332. 
4. Ould-Slimane M, Thong P, Perez A, Roussignol X, Dujardin FH. The role of Intraoperative 3D navigation for pelvic bone tumor resection. Orthop Traumatol Surg Res. 2016;102:807-11.

5. Sabourin M, Biau D, Babinet A, Dumaine V, Tomeno B, Anract P. Surgical management of pelvic primary bone tumors involving the sacroiliac joint. Orthop Traumatol Surg Res. 2009;95:284-92.

6. Deloin X, Dumaine V, Biau D, Karoubi M, Babinet A, Tomeno B, Anract P. Pelvic chondrosarcomas: surgical treatment options. Orthop Traumatol Surg Res. 2009;95:393-401. 7. Workman ML, Bailey DF, Cunningham BL. Popliteal-based filleted lower leg musculocutaneous free-flap coverage of a hemipelvectomy defect. Plast Reconstr Surg. 1992;89:326-329.

8. Sara T, Kour AK, Das De S, Rauff A, Pho RW. Wound cover in a hindquarter amputation with a free flap from the amputated limb. A case report. Clin Orthop. 1994;304:248-251.

9. Yamamoto Y, Minakawa H, Takeda N. Pelvic reconstruction with a free fillet lower leg flap. Plast Reconstr Surg. 1997;99:1439-1441.

10. Yamamoto Y, Sugihara T. Pelvic reconstruction with a free fillet lower leg flap. Plast Reconstr Surg. 2003;111:1475-1476.

11. Ross DA, Lohman RF, Kroll SS, et al. Soft tissue reconstruction following hemipelvectomy. Am J Surg. 1998;176:25-29.

12. Templeton KJ, Toby EB. Free filet leg flap. Clin Orthop. 2001;385:182-185.

13. Morii T, Susa M, Nakayama R, Kishi K, Morioka H, Yabe H. Reconstruction modality based on the spare part concept for massive soft tissue defects following oncological hemipelvectomy. J Orthop Sci. 2009;14:192-197. 
14. Faria JC, Aguiar S, Ferreira F de O, Lopes A. Fillet flap for reconstruction after hemipelvectomy: report of three cases. J Plast Reconstr Aesthet Surg. 2009;62:e110-111. 15. Burd A, Wong KC, Kumta SM. Aggressive surgical palliation for advanced girdle tumours. Indian J Plast Surg. 2012;45:16-21.

16. Bibbo C, Newman AS, Lackman RD, Levin LS, Kovach SJ. A simplified approach to reconstruction of hemipelvectomy defects with lower extremity free fillet flaps to minimize ischemia time. J Plast Reconstr Aesthet Surg. 2015;68:1750-1754

17. Boehmler JH, Francis SH, Grawe RK, Mayerson JL. Reconstruction of an external hemipelvectomy defect with a two-stage fillet of leg-free flap. J Reconstr Microsurg, 2010;26:271-276

18. Enneking WF. A system of staging musculoskeletal neoplasms. Clin Orthop Relat Res, 1986;204: 9-24.

19. Mat Saad AZ, Halim AS, Faisham WI, Azman WS, Zulmi W. Soft tissue reconstruction following hemipelvectomy: eight-year experience and literature review. ScientificWorldJournal. 2012;2012:702904.

20. Knox K, Bitzos I, Granick M, Datiashvili R, Benevenia J, Patterson F. Immediate reconstruction of oncologic hemipelvectomy defects. Ann Plast Surg. 2006;57:184-189. 21. Temple WJ, Mnaymneh W, Ketcham AS. The total thigh and rectus abdominis myocutaneous flap for closure of extensive hemipelvectomy defects. Cancer. 1982;50:25242528.

22. Newsome RE, Warner MA, Wilson SC, Sabeeh VN, Jansen DA, McKee PR. Extracorporeal bypass preserved composite anterior thigh free flap (periosteo-musculo-fasciocutaneous) for hemipelvectomy reconstruction: utilizing the periosteal component for abdominal wall fascial reconstruction. Ann Plast Surg. 2005;54:318-322. 
23. Douglass HO, Razack M, Holyoke ED. Hemipelvectomy. Arch Surg. 1960. $1975 ; 110: 82-85$.

24. Harris GD, Lewis VL, Nagle DJ, Edelson RJ, Kim PS. Free flap reconstruction of the lower back and posterior pelvis: Indications, principles, and techniques. J Reconstr Microsurg. 1988;4:169-178.

25. Kulaylat MN, Froix A, Karakousis CP. Blood supply of hemipelvectomy flaps: the anterior flap hemipelvectomy. Arch Surg. 2001;136:828-831.

26. Chretien PA, Sugarbaker PH. Surgical technique of hemipelvectomy in the lateral position. Surgery. 1981;90:900-909.

27. Frey C, Matthews LS, Benjamin H, Fidler WJ. A new technique for hemipelvectomy. Surg Gynecol Obstet. 1976;143:753-756.

28. Karakousis CP. Hemipelvectomy. In: Atlas of Operations for Soft Tissue Tumors. New York: McGraw-Hill, 1985: 335-350.

29. Ariel IM, Shah JP. The conservative hemipelvectomy. Surg Gynecol Obstet. 1977;144:406-413.

30. Senchenkov A, Moran SL, Petty PM, et al. Predictors of complications and outcomes of external hemipelvectomy wounds: account of 160 consecutive cases. Ann Surg Oncol. $2008 ; 15: 355-363$.

31. Karakousis CP, Vezeridis MP. Variants of hemipelvectomy. Am J Surg. $1983 ; 145: 273-277$.

32. Higinbotham NL, Marcove RC, Casson P. Hemipelvectomy: a clinical study of 100 cases with five-year-follow-up on 60 patients. Surgery. 1966;59:706-708.

33. Apffelstaedt JP, Driscoll DL, Spellman JE, Velez AF, Gibbs JF, Karakousis CP. Complications and outcome of external hemipelvectomy in the management of pelvic tumors. Ann Surg Oncol. 1996;3:304-309. 
34. Luna-Perez P, Herrera L. Medial thigh myocutaneous flap for covering extended hemipelvectomy. Eur J Surg Oncol. 1995;21:623-626.

35. Larson DL, Liang MD. The quadriceps musculocutaneous flap: a reliable, sensate flap for the hemipelvectomy defect. Plast Reconstr Surg. 1983;72:347-354.

36. Dormans JP, Vives M. Wound coverage after modified hip disarticulation using a total adductor myocutaneous flap. Clin Orthop. 1997;335:218-223.

37. Karakousis C, Sugarbaker PH. Sacrectomy. In: Malawer MM, Sugarbaker PH, eds. Musculoskeletal Cancer Surgery: Treatment of Sarcomas and Allied Diseases. Dordrecht, The Netherlands: Kluwer Academic Publishers; 2001: 413-422.

38. Sugarbaker PH, Malawer MM, Henshaw R. Anterior flap hemipelvectomy. In: Malawer MM, Sugarbaker PH, eds. Musculoskeletal Cancer Surgery: Treatment of Sarcomas and Allied Diseases. Dordrecht, The Netherlands: Kluwer Academic Publishers; 2001:305317.

39. Marfori ML, Wang EH. Adductor myocutaneous flap coverage for hip and pelvic disarticulations of sarcomas with buttock contamination. Clin Orthop. 2011;469:257-263. 40. Taylor GI, Corlett R, Boyd JB. The extended deep inferior epigastric flap: a clinical technique. Plast Reconstr Surg. 1983;72:751-765.

41. Horch RE, Gitsch G, Schultze-Seemann W. Bilateral pedicled myocutaneous vertical rectus abdominus muscle flaps to close vesicovaginal and pouch-vaginal fistulas with simultaneous vaginal and perineal reconstruction in irradiated pelvic wounds. Urology. 2002;60:502-507.

42. Muramatsu K, Ihara K, Ooi R, Imazyo Y, Taguchi T. Experiences with the "reverse" latissimus dorsi myocutaneous flap. Plast Reconstr Surg. 2006;117:2456-2459.

43. Chandrasekhar B, Sloan GM, Beatty JD. The external oblique myocutaneous flap for extended hemipelvectomy reconstruction. Cancer. 1988;62:1022-1025. 
44. O'Brien BM, Barton RM, Pribaz JJ. The wrist as an immediate free flap carrier for reconstruction of the pelvis; a case report. Br J Plast Surg. 1987;40:427-431.

45. Butler CE. Reconstruction of an extensive hemipelvectomy defect using a pedicled upper and lower leg in-continuity fillet flap. Plast Reconstr Surg. 2002;109:1060-1065.

46. Cavadas PC, Landín L. Traumatic complete hemipelvectomy treated with a free fillet flap. J Trauma. 2008;65:1551-1553.

47. Conrad EU. Pitfalls in diagnosis: pediatric musculoskeletal tumors. Pediatr Ann. 1989;18:45-7, 50-52. 
Figure 1. Patient cas $\mathrm{n}^{\circ}$ 4, tableau 2. a. Coupe frontale TDM objectivant l'envahissement local d'un chondrosarcome. b. Coupe axiale TDM avec l'inclusion tumorale de l'artère iliaque externe $(\leftarrow)$.

Figure 2. Installation du patient.

Figure 3. Dessin cutané de l'abord du lambeau de jambe totale.

Figure 4a. Lambeau de jambe totale avec fibula non dépériostée. b. Vue per-opératoire du membre inférieur après la levée du lambeau (* : chondrosarcome).

Figure 5a. Radiographie post-opératoire d'une désarticulation inter-ilio abdominale gauche. b. Radiographie, à 4 mois post-opératoire, objectivant une ossification entre la symphyse pubienne et la fibula.

Figure 6. TDM, à quatre mois post-opératoires, mettant en évidence une ossification entre la symphyse pubienne et la fibula. a. Vue antérieure. b. Vue postérieure.

Figure 7. Aspect clinique du lambeau à 8 mois post-opératoires.

Vidéo 1. Marche d'un patient avec prothèse canadienne. 
Tableau 1 : Caractéristiques individuelles des patients. 


\begin{tabular}{|c|c|c|c|c|c|c|}
\hline & $\begin{array}{l}\text { Age } \\
\text { Sexe }\end{array}$ & Diagnostic & Origine & Envahissement & $\begin{array}{l}\text { Classification } \\
\text { Enneking [18] }\end{array}$ & $\begin{array}{l}\text { Antécédent de chirurgie locale et } \\
\text { traitement néo-adjuvant }\end{array}$ \\
\hline Cas 1 & $\begin{array}{l}61 \text { ans } \\
\text { Masculin }\end{array}$ & $\begin{array}{l}\text { Ostéosarcome de haut grade, } \\
\text { variante fibroblastique }\end{array}$ & Fémur proximal gauche & $\begin{array}{l}\text { Envahissement circonférentiel du } \\
\text { fémur, du moyen fessier et des } \\
\text { vaisseaux fémoraux }\end{array}$ & Stade II B & $\begin{array}{l}\text { - Ostéosynthèse par enclouage centro- } \\
\text { médullaire sur fracture pathologique } \\
\text { - Chimiothérapie néo-adjuvante }\end{array}$ \\
\hline Cas 2 & $\begin{array}{l}32 \text { ans } \\
\text { Masculin }\end{array}$ & Sarcome à cellules claires & Racine de cuisse droite & $\begin{array}{l}\text { Envahissement circonférentiel des } \\
\text { parties molles de la fesse et des } \\
\text { vaisseaux iliaques }\end{array}$ & Stade II B & Aucun \\
\hline Cas 3 & $\begin{array}{l}61 \text { ans } \\
\text { Masculin }\end{array}$ & $\begin{array}{l}\text { Chondrosarcome très } \\
\text { majoritairement de grade } 1 \text { et } \\
\text { focalement de grade } 2\end{array}$ & $\begin{array}{l}\text { Cadre obturateur } \\
\text { gauche }\end{array}$ & $\begin{array}{l}\text { Envahissement de l'articulation coxo- } \\
\text { fémorale, des muscles pelvi- } \\
\text { trochantériens, des muscles adducteurs } \\
\text { et de l'artère fémorale profonde }\end{array}$ & Stade II B & Aucun \\
\hline Cas 4 & $\begin{array}{l}56 \text { ans } \\
\text { Féminin }\end{array}$ & Chondrosarcome grade 2 & $\begin{array}{l}\text { Branche ilio-pubienne } \\
\text { gauche }\end{array}$ & $\begin{array}{l}\text { Envahissement endopelvien à partir du } \\
\text { Scarpa, infiltration de la paroi latérale } \\
\text { de la vessie et envahissement des } \\
\text { vaisseaux iliaques et fémoraux }\end{array}$ & Stade II B & $\begin{array}{l}\text { Résection de la branches ilio-pubienne } \\
\text { gauche pour chondrosarcome de bas } \\
\text { grade }(j+10 \text { ans }) \text { et résection du bord } \\
\text { antérieur de l'aile iliaque pour } \\
\text { chondrosarcome grade } 2(j+3 \text { ans })\end{array}$ \\
\hline Cas 5 & $\begin{array}{l}62 \text { ans } \\
\text { Masculin }\end{array}$ & $\begin{array}{l}\text { Chondrosarcome à cellules } \\
\text { claires }\end{array}$ & Fémur proximal gauche & $\begin{array}{l}\text { Envahissement des parties molles } \\
\text { glutéales et de la racine de cuisse ; et } \\
\text { envahissement vasculaire }\end{array}$ & Stade II B & $\begin{array}{l}\text { Résection et PTH pour chondrosarcome } \\
\text { à cellules claires }(j+22 \text { ans }) . \\
\text { Changement PTH }(j+5 \text { ans })\end{array}$ \\
\hline Cas 6 & $\begin{array}{l}50 \text { ans } \\
\text { Masculin }\end{array}$ & $\begin{array}{l}\text { Ostéosarcome secondaire sur } \\
\text { dysplasie fibreuse }\end{array}$ & Fémur proximal gauche & $\begin{array}{l}\text { Envahissement des parties molles de la } \\
\text { fesse, de la cuisse gauche et } \\
\text { envahissement vasculaire }\end{array}$ & Stade II B & $\begin{array}{l}\text { - Ostéosynthèse sur fracture } \\
\text { pathologique } \\
\text { - Chimiothérapie néo-adjuvante }\end{array}$ \\
\hline Cas 7 & $\begin{array}{l}59 \text { ans } \\
\text { Masculin }\end{array}$ & $\begin{array}{l}\text { Sarcome inclassé de haut } \\
\text { grade à cellules fusiformes }\end{array}$ & Fémur proximal droit & $\begin{array}{l}\text { Pas d'envahissement mais } \\
\text { contamination coxo-fémorale } \\
\text { iatrogénique }\end{array}$ & Stade II B & $\begin{array}{l}\text { PTH }(j+12 \text { ans }) \text {. Changement PTH sur } \\
\text { fracture pathologique }(j+7 \text { mois })\end{array}$ \\
\hline
\end{tabular}

PTH : prothèse totale de hanche 
Tableau 2 : Techniques chirurgicales, complications des lambeaux et suivi.

\begin{tabular}{|c|c|c|c|c|c|c|c|c|}
\hline & Chirurgie & Chronologie & Lambeau & Anastomoses & $\begin{array}{l}\text { Durée } \\
\text { d'ischémie }\end{array}$ & $\begin{array}{l}\text { Complications du } \\
\text { lambeau }\end{array}$ & $\begin{array}{l}\text { Traitement } \\
\text { complémentaire } \\
\text { post-chirurgie }\end{array}$ & Survie et suivi \\
\hline Cas 1 & DIA gauche & $\begin{array}{l}\text { Amputation puis dissection } \\
\text { et levée du lambeau sur } \\
\text { table puis anastomoses }\end{array}$ & $\begin{array}{l}\text { Loge postérieure } \\
\text { superficielle de } \\
\text { jambe }\end{array}$ & $\begin{array}{l}\text { Termino-terminales entre les } \\
\text { pédicules iliaque externe et } \\
\text { fémoral }\end{array}$ & $-\mathrm{NC}$ & $\begin{array}{l}\text { - Thrombose de la } \\
\text { veine } \\
\text { - Infection locale }\end{array}$ & Aucun & $\begin{array}{l}\text { - Décédé des suites } \\
\text { de la maladie } \\
\text { - } 17 \text { mois de suivi }\end{array}$ \\
\hline Cas 2 & DIA droite & $\begin{array}{l}\text { Amputation puis dissection } \\
\text { et levée du lambeau sur } \\
\text { table puis anastomoses }\end{array}$ & $\begin{array}{l}\text { Loge postérieure } \\
\text { superficielle de } \\
\text { jambe }\end{array}$ & $\begin{array}{l}\text { - Termino-terminale entre } \\
\text { l'artère iliaque primitive et } \\
\text { l'artère fémorale } \\
\text { - Termino-latérale entre le } \\
\text { carrefour ilio-cave et la veine } \\
\text { fémorale }\end{array}$ & $-\mathrm{NC}$ & $\begin{array}{l}\text { Petite zone } \\
\text { nécrotique }\end{array}$ & Aucun & $\begin{array}{l}\text { - Décédé des suites } \\
\text { de la maladie } \\
\text { - } 6,5 \text { mois de suivi }\end{array}$ \\
\hline Cas 3 & DIA gauche & $\begin{array}{l}\text { Double équipe : abord } \\
\text { pelvien et dissection du } \\
\text { lambeau en parallèle puis } \\
\text { amputation et levée du } \\
\text { lambeau et anastomoses }\end{array}$ & $\begin{array}{l}\text { Jambe avec fibula } \\
\text { non dépériostée }\end{array}$ & $\begin{array}{l}\text { Termino-latérales entre les } \\
\text { pédicules iliaque primitif et } \\
\text { fémoral }\end{array}$ & $70 \mathrm{~min}$ & Aucune & Aucun & $\begin{array}{l}\text { - En vie, sans } \\
\text { récidive ni } \\
\text { métastase } \\
-8 \text { mois de suivi }\end{array}$ \\
\hline Cas 4 & DIA gauche & $\begin{array}{l}\text { Double équipe : abord } \\
\text { pelvien et dissection du } \\
\text { lambeau en parallèle puis } \\
\text { amputation et levée du } \\
\text { lambeau et anastomoses }\end{array}$ & $\begin{array}{l}\text { Jambe avec fibula } \\
\text { non dépériostée }\end{array}$ & $\begin{array}{l}\text { Termino-latérales entre les } \\
\text { pédicules iliaque primitif et } \\
\text { fémoral }\end{array}$ & $75 \mathrm{~min}$ & Fistule de la fibula & Aucun & $\begin{array}{l}\text { - En vie, sans } \\
\text { récidive ni } \\
\text { métastase } \\
\text { - } 12 \text { mois de suivi }\end{array}$ \\
\hline Cas 5 & DIA gauche & $\begin{array}{l}\text { Dissection du lambeau, } \\
\text { amputation puis levée du } \\
\text { lambeau et anastomoses }\end{array}$ & $\begin{array}{l}\text { Jambe avec fibula } \\
\text { non dépériostée }\end{array}$ & $\begin{array}{l}\text { - Termino-latérale entre } \\
\text { l'artère iliaque externe et } \\
\text { l'artère fémorale } \\
\text { - Termino-latérale entre la } \\
\text { veine iliaque commune et la } \\
\text { veine fémorale }\end{array}$ & $-\mathrm{NC}$ & $\begin{array}{l}\text { Défaut de suture } \\
\text { de l'anastomose } \\
\text { veineuse }\end{array}$ & Aucun & $\begin{array}{l}\text { - Décédé des suites } \\
\text { de la maladie } \\
\text { - } 7 \text { mois de suivi }\end{array}$ \\
\hline Cas 6 & $\begin{array}{l}\text { DCF gauche avec } \\
\text { résection de la région } \\
\text { glutéale }\end{array}$ & $\begin{array}{l}\text { Dissection du lambeau, } \\
\text { amputation puis levée du } \\
\text { lambeau et anastomoses }\end{array}$ & $\begin{array}{l}\text { Loge postérieure } \\
\text { superficielle de } \\
\text { jambe }\end{array}$ & $\begin{array}{l}\text { Termino-latérales entre les } \\
\text { pédicules iliaque externe et } \\
\text { fémoral }\end{array}$ & $-\mathrm{NC}$ & Infection locale & Aucun & $\begin{array}{l}\text { - Décédé des suites } \\
\text { de la maladie } \\
\text { - } 21 \text { mois de suivi }\end{array}$ \\
\hline Cas 7 & DIA droite & $\begin{array}{l}\text { Dissection du lambeau, } \\
\text { amputation puis levée du } \\
\text { lambeau et anastomoses }\end{array}$ & $\begin{array}{l}\text { Jambe avec fibula } \\
\text { non dépériostée }\end{array}$ & $\begin{array}{l}\text { - Termino-latérale entre } \\
\text { l'artère iliaque externe et } \\
\text { l'artère fémorale }\end{array}$ & $130 \mathrm{~min}$ & $\begin{array}{l}\text { Echec, perte du } \\
\text { lambeau sur } \\
\text { thrombose }\end{array}$ & $\begin{array}{l}\text { Multiples } \\
\text { chimiothérapies }\end{array}$ & $\begin{array}{l}\text { - Décédé des suites } \\
\text { de la maladie } \\
\text { - } 19 \text { mois de suivi }\end{array}$ \\
\hline
\end{tabular}




\begin{tabular}{|l|l|l|l|l|l|l|l|}
\hline & & & $\begin{array}{l}\text { - Termino-latérale entre la } \\
\text { veine iliaque externe et la } \\
\text { veine fémorale }\end{array}$ & & & & \\
\hline
\end{tabular}

DIA : désarticulation inter-ilio-abdominale, DCF : désarticulation coxofémorale, NC : non connu 
Tableau 3 : Articles rapportant 16 cas d'hémipelvectomie avec reconstruction par lambeau libre de jambe.

\begin{tabular}{|c|c|c|c|c|c|c|}
\hline Article & Age & Diagnostic & Lambeau & Anastomoses & Complications & $\begin{array}{l}\text { Marche/Appareil } \\
\text { lage }\end{array}$ \\
\hline Workman et al. [7] & 33 ans & Hystiocytome fibreux & $\begin{array}{l}\text { Jambe avec fibula } \\
\text { dépériostée }\end{array}$ & $\begin{array}{l}\text { Pédicule iliaque et pédicule } \\
\text { fémoral }\end{array}$ & Aucune & $\mathrm{NC}$ \\
\hline Sara et al. [8] & 28 ans & Angiosarcome & $\begin{array}{l}\text { Jambe avec fibula } \\
\text { dépériostée }\end{array}$ & $\begin{array}{l}\text { - Artère glutéale supérieure } \\
\text { et artère tibiale postérieure } \\
\text { - Artère épigastrique } \\
\text { inférieure et artère tibiale } \\
\text { antérieure }\end{array}$ & $10 \%$ nécrose & $\mathrm{NC}$ \\
\hline Yamamoto et al. [9] & 55 ans & Chondrosarcome & $\begin{array}{l}\text { Jambe avec fibula } \\
\text { dépériostée }\end{array}$ & $\begin{array}{l}\text { Pédicule iliaque interne et } \\
\text { pédicule fémoral }\end{array}$ & Aucune & $\begin{array}{l}\text { Marche avec } \\
\text { prothèse }\end{array}$ \\
\hline Yamamoto et al. [10] & $\mathrm{NC}$ & Chondrosarcome & $\begin{array}{l}\text { Jambe avec fibula non } \\
\text { dépériostée }\end{array}$ & $\mathrm{NC}$ & Aucun & $\begin{array}{l}\text { Marche avec } \\
\text { prothèse }\end{array}$ \\
\hline Ross et al. [11] & $\mathrm{NC}$ & Tumeur maligne & $\begin{array}{l}\text { Jambe avec fibula } \\
\text { dépériostée }\end{array}$ & $\begin{array}{l}\text { Pédicule fémorale } \\
\text { superficielle controlatéral et } \\
\text { pédicule fémoral }\end{array}$ & Aucune & $\mathrm{NC}$ \\
\hline Templeton et al. [12] & 73 ans & Chondrosarcome & $\begin{array}{l}\text { Jambe avec fibula non } \\
\text { dépériostée }\end{array}$ & $\begin{array}{l}\text { Pédicule iliaque externe et } \\
\text { pédicule fémoral }\end{array}$ & Aucune & $\begin{array}{l}\text { Pas de prothèse } \\
\text { mais } \\
\text { déambulateur }\end{array}$ \\
\hline Morii et al. [13] & $\begin{array}{l}-52 \text { ans } \\
-41 \text { ans }\end{array}$ & $\begin{array}{l}\text { - Carcinome épidermoïde } \\
\text { - Chondrosarcome }\end{array}$ & $\begin{array}{l}\text { Jambe avec fibula } \\
\text { dépériostée }\end{array}$ & $\begin{array}{l}\text { Pédicule iliaque interne et } \\
\text { pédicule fémoral }\end{array}$ & Aucune & $\mathrm{NC}$ \\
\hline
\end{tabular}




\begin{tabular}{|c|c|c|c|c|c|c|}
\hline Faria et al. [14] & $\begin{array}{l}-32 \text { ans } \\
-42 \text { ans } \\
-43 \text { ans }\end{array}$ & $\begin{array}{l}\text { - Fibrohistiocytome malin } \\
\text { - Sarcome pléomorphe haut grade } \\
\text { - Liposarcome myxoïde }\end{array}$ & $\begin{array}{l}\text { Jambe avec fibula } \\
\text { dépériostée }\end{array}$ & $\begin{array}{l}\text { Pédicule iliaque et pédicule } \\
\text { fémoral }\end{array}$ & Aucune & $\begin{array}{l}1 / 3 \text { marche avec } \\
\text { béquilles }\end{array}$ \\
\hline Andrex Burd et al. [15] & 37 ans & Sarcome épithéloïde & $\begin{array}{l}\text { Jambe avec fibula } \\
\text { dépériostée }\end{array}$ & $\mathrm{NC}$ & Aucune & $\begin{array}{l}\text { Marche avec } \\
\text { déambulateur }\end{array}$ \\
\hline Bibbo et al. [16] & $\begin{array}{l}-15 \text { ans } \\
-45 \text { ans } \\
-50 \text { ans }\end{array}$ & $\begin{array}{l}\text { - Ostéosarcome } \\
\text { - Léiomyosarcome } \\
\text { - Chondrosarcome }\end{array}$ & $\begin{array}{l}\text { - Jambe avec fibula } \\
\text { dépériostée } \\
\text { - Jambe avec fibula } \\
\text { dépériostée } \\
\text { - Jambe avec fibula } \\
\text { dépériostée et cuisse } \\
\text { distale }\end{array}$ & $\begin{array}{l}\text { - Pédicule iliaque externe et } \\
\text { pédicule poplité } \\
\text { - Pédicule iliaque externe et } \\
\text { pédicule poplité } \\
\text { - Pédicule iliaque externe et } \\
\text { pédicule fémoral superficiel }\end{array}$ & $\begin{array}{l}\text { - Aucune } \\
\text { - Aucune } \\
\text { - Aucune }\end{array}$ & $\begin{array}{l}-\mathrm{NC} \\
-\mathrm{NC} \\
-\mathrm{NC}\end{array}$ \\
\hline Boehmler et al. [17] & -55 ans & - Sarcomes radio-induit & $\begin{array}{l}\text { - Jambra avec fibula } \\
\text { dépériostée }\end{array}$ & $\begin{array}{l}\text { - Pédicule fémoral } \\
\text { superficiel controlatéral ( } 1 \\
\text { artère et } 2 \text { veines) et } \\
\text { pédicule poplité }\end{array}$ & $\begin{array}{l}\text { - Zone de } \\
\text { désunion } \\
\text { cicatricielle : } \\
\text { parage } \\
\text { chirurgical à } 21 \\
\text { jours }\end{array}$ & $\begin{array}{l}\text { Marche avec } \\
\text { déambulateur }\end{array}$ \\
\hline
\end{tabular}

NC : non connu 


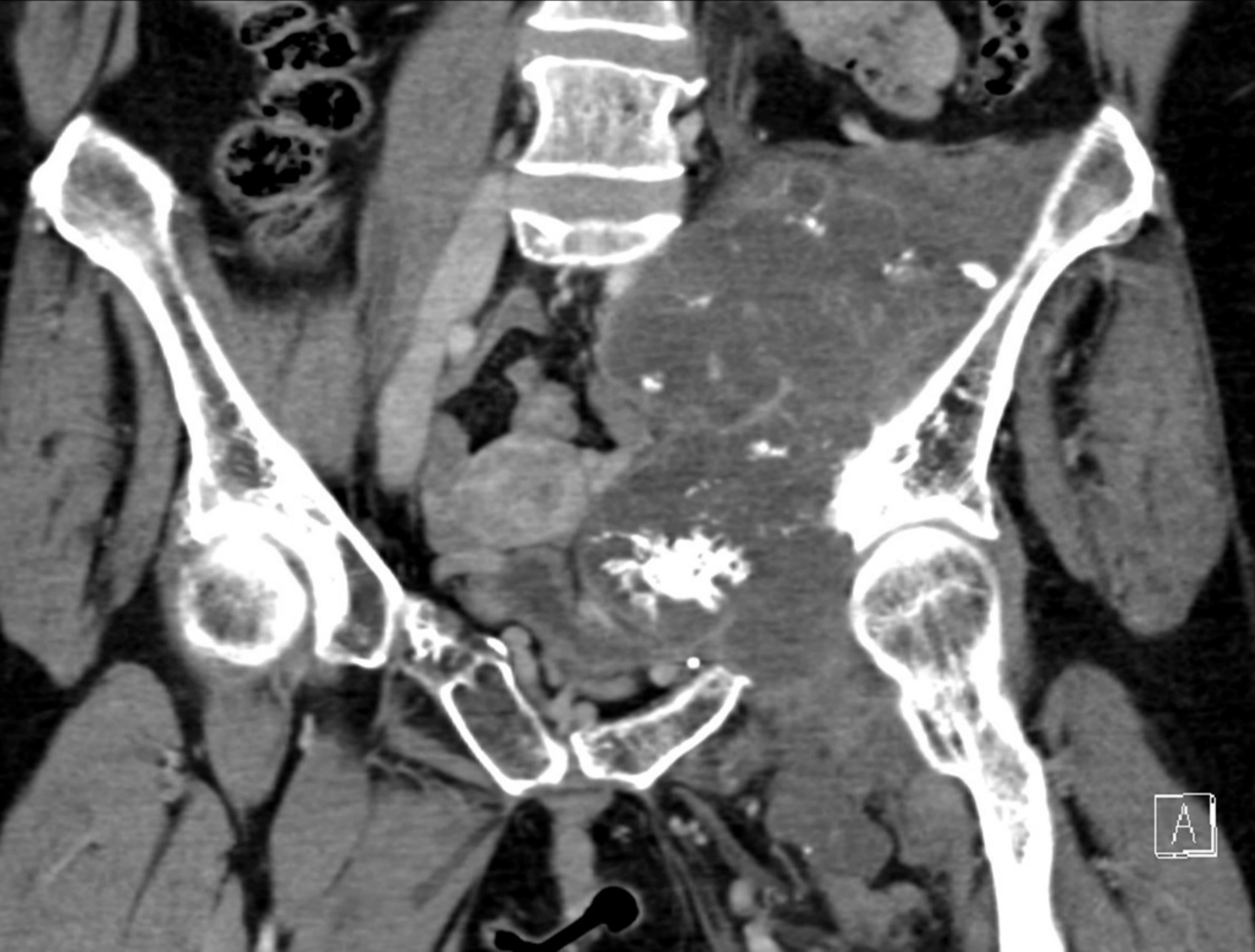




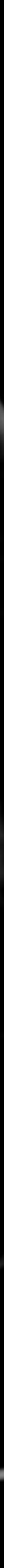




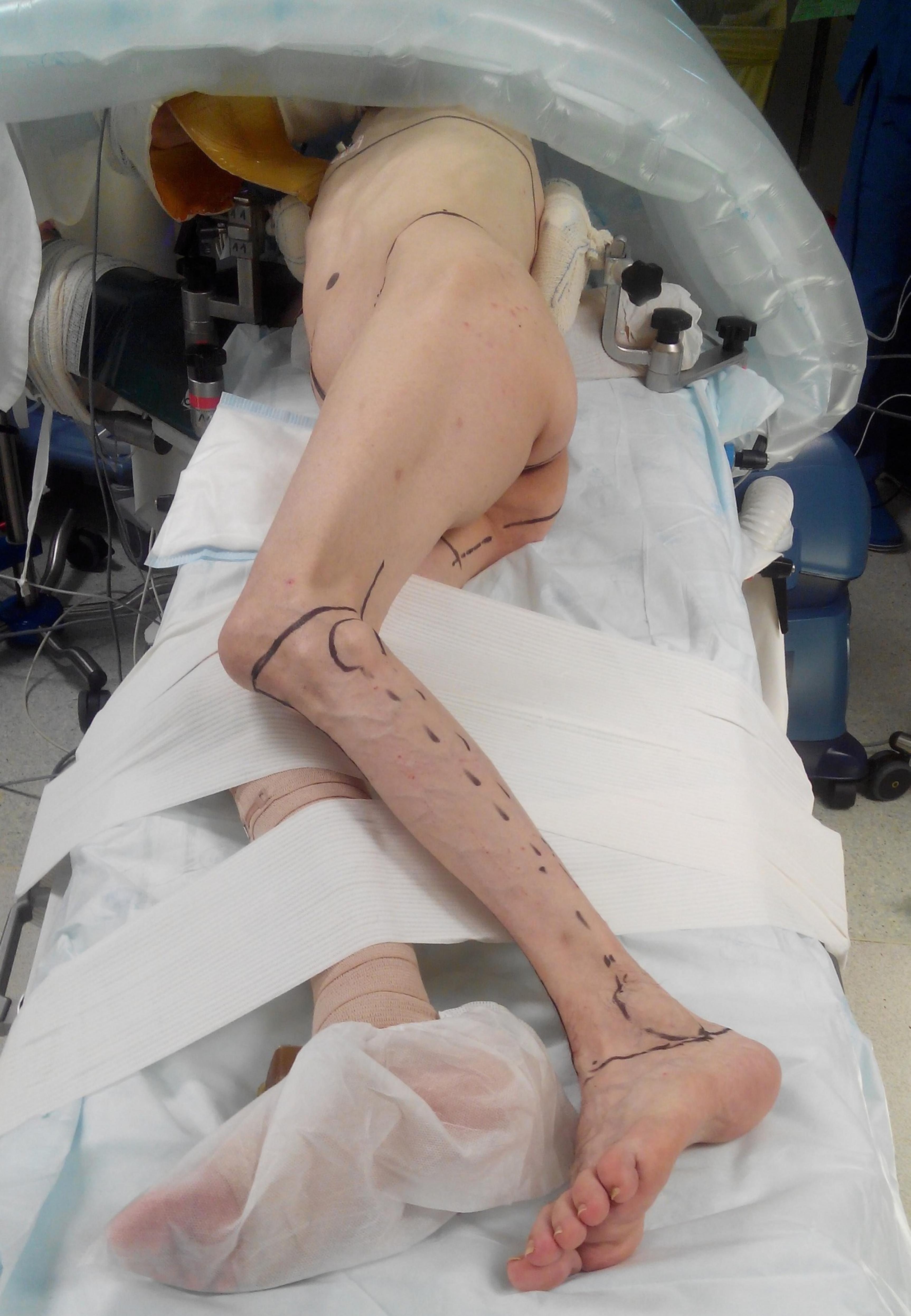




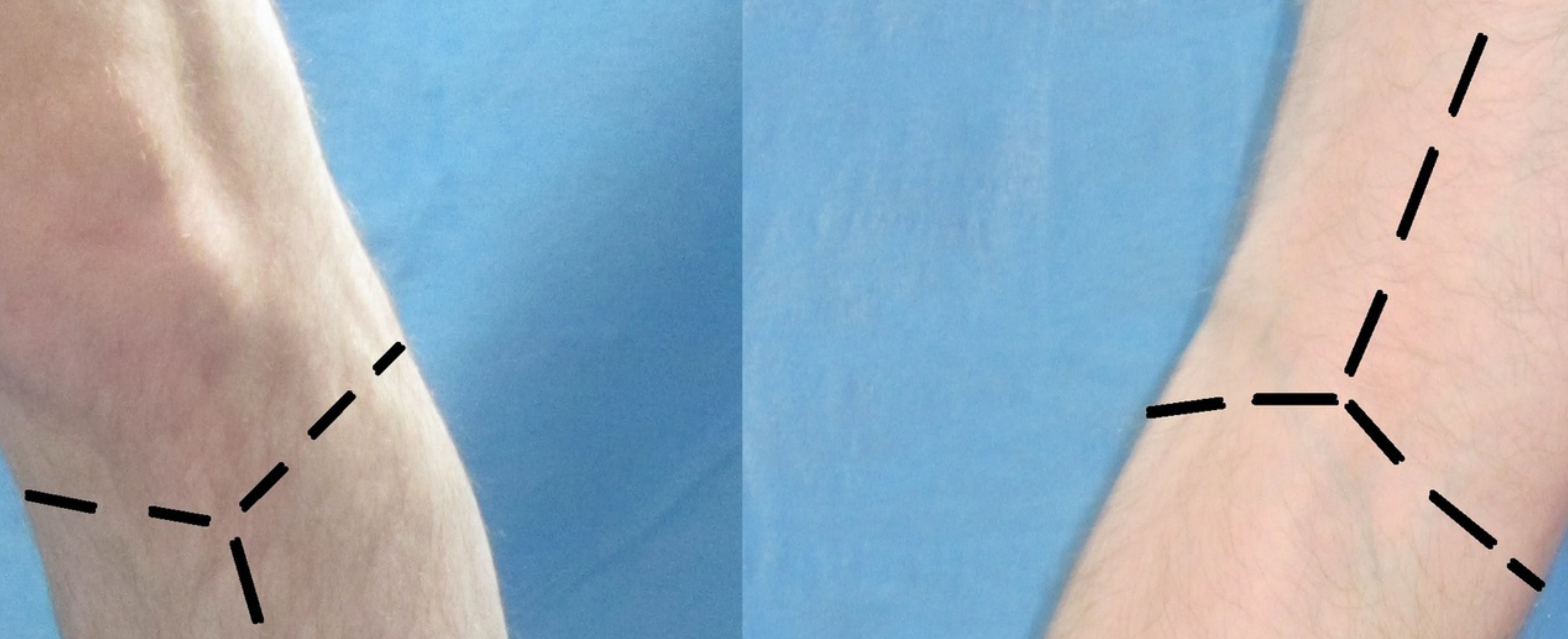

1

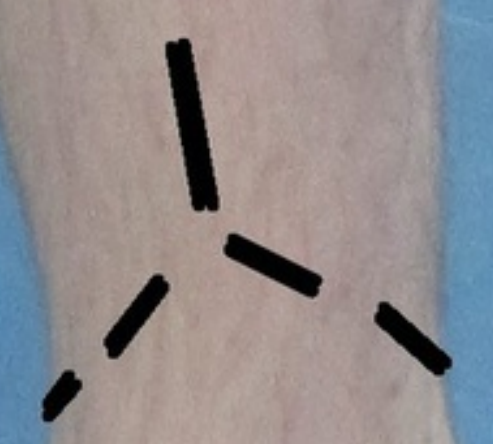



*

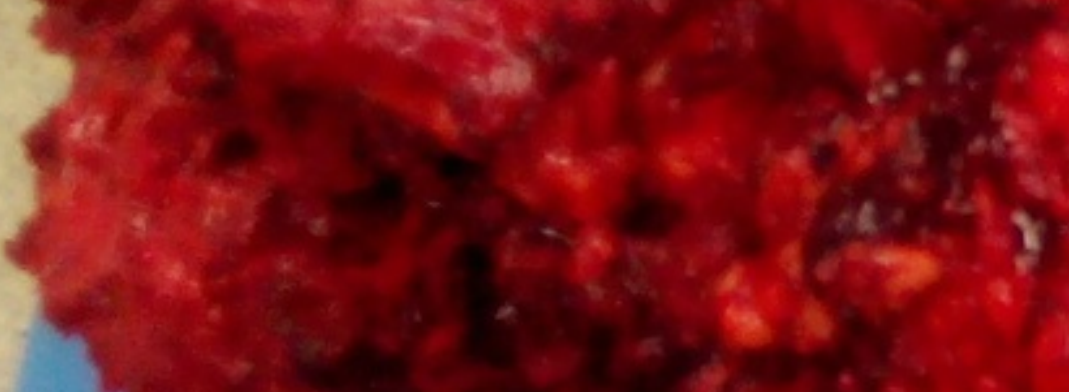

13

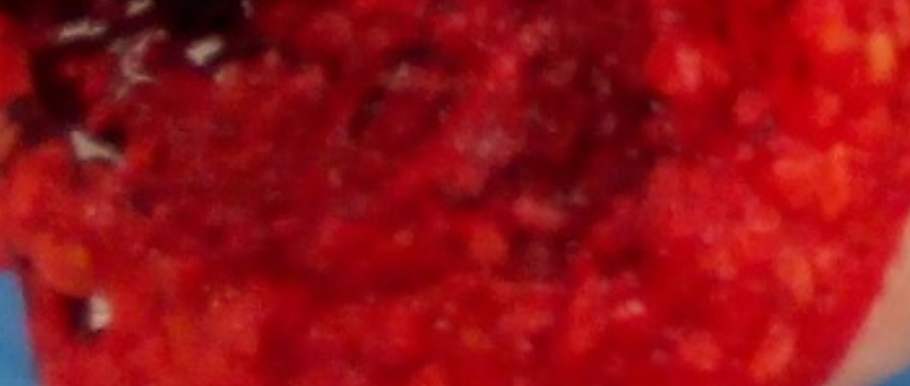




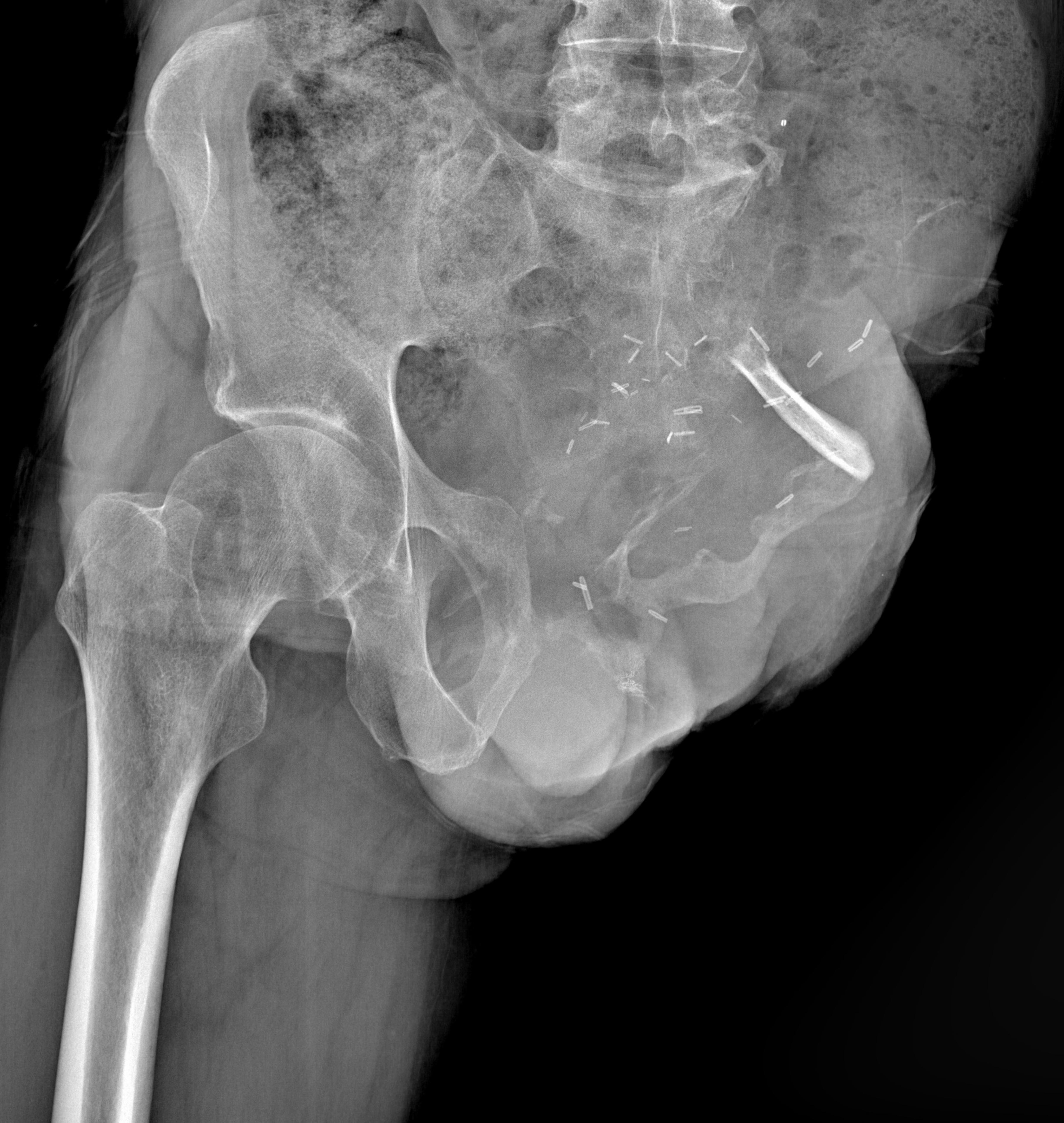




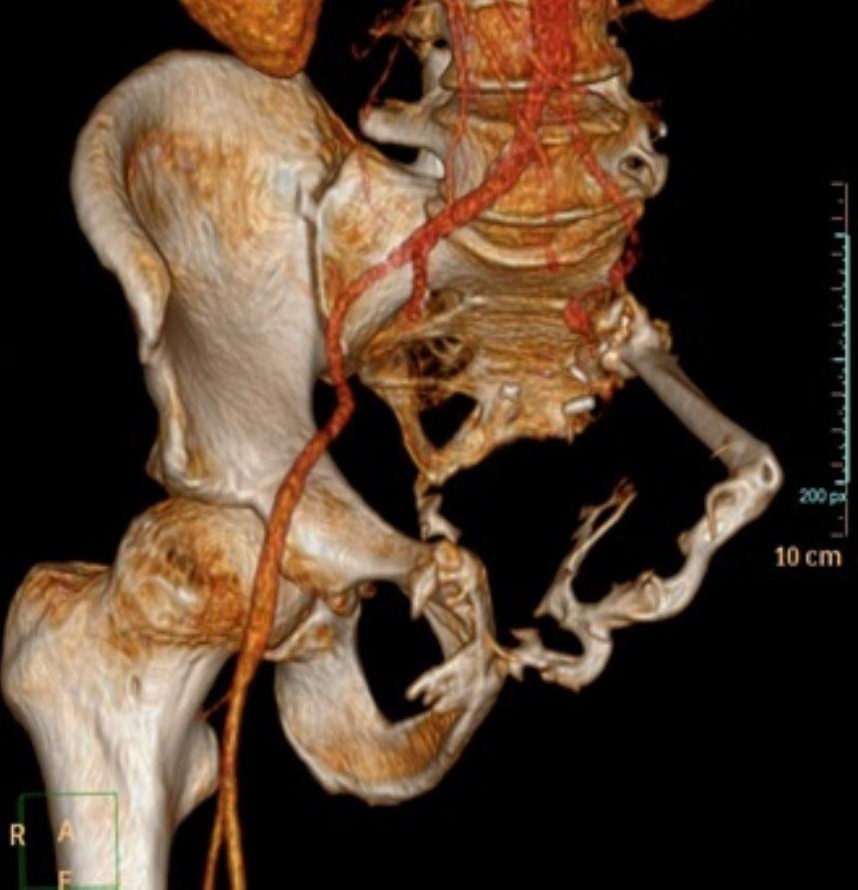




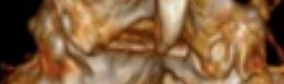

(1) 19:

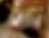

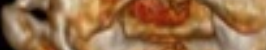

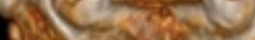

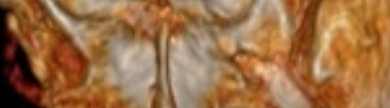

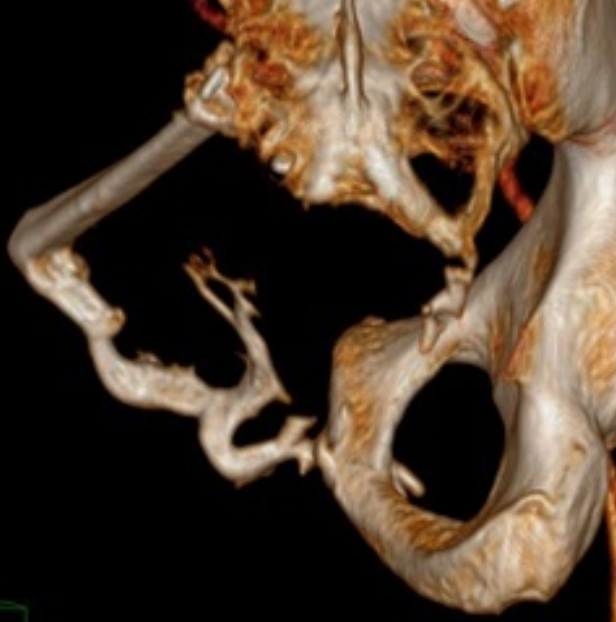


